\title{
Impact of Globalization on Brahui language
}

\author{
Noreen Bano ${ }^{1}$, Dr. Liaqat $\operatorname{Sani}^{2} \&$ Panah Baloch $^{3}$ \\ ${ }^{1 .}$ M: Phil Scholar department of Brahui University of Balochistan. \\ ${ }^{2}$. Associate professor department of Brahui University of Balochistan. \\ ${ }^{3}$. Assistant director Balochistan Agriculture R\&D Center.
}

\begin{abstract}
Brahui is one of the oldest languages of the Sub-continent and Brahui speaking people are found in Balochistan, Sindh, Afghanistan, Turkmenistan and Iran. Some researchers claimed that Brahui is an Aryan language others asserted that it is a Turko-Iranian language. Another group of intellectuals also emphasize that the traces of Brahui language have been found in the remainants of Mohenjo Daro and it has Dravidian origin and same language is being spoken in some parts of India and Sri Lanka. According to historians Brahui's and Baloch's belong to same race. Balochi speaking people entered Mekran while Brahui speaking people entered from Chagai as they defeated the Dravidian rulers of Kalat and entered into matrimonial relationship with the Dravidian and Balochi languages were mixed up and a new language of "Brahui" was born due to same relationship. According to reports about four million people of the above mentioned countries and regions speak Brahui language. Languages are vehicles of our culture, collective memory and values. They are an essential component of our identities, and a building block of our diversity and living heritage. Globalized economics and media are altering the face of culture around the globe, reducing the number of languages that human's converse. As the world economy becomes more integrated, a common tongue has become more important than ever to promote commerce, and that puts speakers of regional dialects and minority languages at a distinct disadvantage. In addition, information technology has pressured languages to become more standardized, further squeezing local variations of language. These pressures are inducing a rapid die-off of languages around the world. UNESCO Atlas of the World's Languages in Danger declaring that Brahui is vulnerable language. A small scale print and electronic media are playing important role in the promotion of Brahui language but have no ample capacity to stop the invasion of globalization. The findings reveal that some Brahui words, names of boys and girls, days, relationships, terminologies, names of food items are replaced by the words of the other dominant languages of electronic media.
\end{abstract}

Keywords: Globalization, Languages. Culture, identities, UNESCO, Brahui language 


\section{Background}

Brahui is one of the oldest languages of the Indian Sub-continent and Brahui speaking people are found in Balochistan, Sindh, Afghanistan, Turkmenistan and Iran. There are many theories about the origin of Brahui language. Many people claimed that Brahui is an Aryan language others say that it is a Turko-Iranian language. A few Intellectuals articulate that the traces of Brahui language have been found in the remainants of Mohenjo Daro, and it belonged Dravidian origin and same language is being spoken in some parts of India and Sri Lanka. According some historians Brahui's and Baloch's belong to same race. Balochi speaking people entered Mekran while Brahui speaking people entered from Chagai as they conquered the Dravidian rulers of Kalat and entered into matrimonial relationship with the Dravidian and Balochi languages were mixed up and a new language of "Brahui" was born due to same relationship (www.brahvilanguage.com). According the census reports of 1901 population of Brahui speaking persons was 300000.00 , in 1911 increased as $167,787.00$, in 1931 was 224,415.00 in 1951 was $262,463.00$ and in 1961 was $365,772.00$. Some described that there are 2.20 million people from Pakistan and all around world speaks Brahui language (Brahui A.R: 2012: 37). However, 2013 edition of Ethnoloque reports that there are approximately 4.2 million speakers; 4 million live in Pakistan mainly in the province of Balochistan.

There are many opinions about the origin of Brahui language and researchers, scholars and linguistics are divided in three major groups. T. Brozow, MB Aimenio, Denis Bray, GR Greisens, Dr. Ernest Trump, G.U Pope, MS Andronov and Bishop Coldwell are first group describe that the Brahui language as Dravidian language. However, Prof. Abdullah Jan Jamaldini, Mir Gul Khan Naseer, Agha Naseer Khan Ahmadzai, Mir Aqil Khan Mengal, Dr. Abdul Razzaq Sabir, Prof. Aziz Mengal and Asser Abdul Qadir Shahwani declared that the Brahui as Aryan language and Agha Naseer Khan Ahmadzai, Mir Aqil Khan Mengal, Dr. Abdul Razzaq Sabir are presumes that it may be Turko-Iranian language (Akhtar Javed: 2007: 20) . 
Renowned linguists and historians Mir Gul Khan Naseer, Agha Naseer Khan Ahmadzai, Mir Aqil Khan Mengal, Malik Saleh Muhammad Lehri and Mir Khuda Bakhsh Marri had acquired the idea from the books of some Arab geographers, historians, and tourists who claim that Brahui belongs to Turko Aryan (Turani) language family and Scythian, Babli and Meds also members of the same family of language. Prof. Javed Akhtar emphasizes that Brahui belongs to Dravidian race and their language was Dravidian, and also asserted that the traces of history of Brahui's is findable in the modern Hijri years. Brahui detached from Proto or old Dravidian race about 4000 years ago. They belonged to Carpentaria Variety branch of Australian race. They entered into subcontinent through Turkamenia and Aral Sea. However, Mir Gul Khan Naseer and Malik Saleh Muhammad Lehri are of the view that Brahui belongs to the clan of those Baloch's who migrated earlier than other Baloch clans. They were settled near the mountainous ranges of "Al-Burz" accordingly, "Al-Burz" was renamed as "Burz Kohi” and with span of time same changed into "Brahui or Barohi". There are three recognized dialects of Brahui which called Jhalwani, Sarawani \& Rakhsani dialect.

\section{Globalization \& languages}

There are many meaning and introduction of the globalization, which are available in different books and dictionaries. Some scholars define that "Globalization is process of interaction and integration among the people, companies, and governments of different nations. It has been defined by different researchers and authors as: Globalization (or globalization) is the process of international integration arising from the interchange of world views, products, ideas and other aspects of culture" (https://.en.wikipedia.org/wiki/Globalization). Globalization is a name of an integrated system and it is speedy mode of communication, reduction of taxes, fastest flow of the world capital, expansion of products of multinational companies, reach of people and it is the idea which aided with information technology (Rehman T. 2004:17). Globalization is a process of interaction and integration among the people, companies, and governments of different 
nations. It is a process driven by international trade and investment and aided by information technology. This is where the problems with globalization begin to arise. Globalization is "the growing interdependence of countries worldwide through the increasing volume and variety of cross-border transaction in goods and services and of international capitals flows, and through the more rapid and widespread diffusion of technology.

Languages are vehicles of our cultures, collective memory and values. They are an essential component of our identities, and a building block of our diversity and living heritage (http://unesdoc.unesco.org/images/0019/001924/192416e.pdf). Promotion and protection of local languages and culture is under discussion in the Pakistan with reference to globalization. It is recognized that the multinational companies and international monitory organization are strengthening global political, economical, and cultural dominancy in the name of development, economical sustainability and prevention of poverty. Due to this disparity is raising high between the poor and wealthy segments of the society in the deprived and backward countries and social values are being disrupted, cultural partition is rising high and people's mentality, material and cultural assets are under pressure of decolonization. In the globalization process the important phenomenon of the human life i.e., mother languages, local cultures and social relations are under threat (Mazhar: 2004:39). Language is valuable asset of the mankind. Moreover, human beings mental, cultural, and spiritual heritage belongs to the language (Siddiqi Khalil: 2000:78). Humans can not start speaking by birth, as they are also like other animals. Furthermore, man slowly copied the birds and other animals and according to their demand of nature started some accents in the shape of words, which became unique-eleven thousands years ago (Marri SM.:2014:25/26). Globalization is a challenge before us. Are we ready to face it? There is a need of constant and absolute struggle, which can provide an ideal environment in a society, where everyone has their basic right to seek knowledge without any fear of losing their languages through educational and literary creations (Mubarak A Dr. 2004: 16). There is small 
scale media i.e., once daily, some weekly, some monthly, countable size fortnightly, quarterly papers, magazine and journals and few hour's programs at PTV Bolan \& Pakistan Broadcasting Corporation and Vsh TV are playing significant role in the encouragement of Brahui language but having no sufficient capacity to stop the influx of globalization. In 2006, UNESCO passed an International treaty to" preserve the rich diversity of the world's means of cultural expression from the dangers of globalization, including its many languages" (TOMUNGeneral_Assembly_Topic.pdf). UNESCO has conducted a survey on 3000 dying languages of the world, and UNESCO Atlas of the World's Languages, declared Brahui in the list of the in danger and vulnerable languages.

\section{Research findings}

In this modernized era, insufficient investigations are conducted to check the impact of globalization on Brahui language. The author took the challenge to document the impacts of globalization on Brahui language and its affect on the various spheres of life. The thematic concerns are of this study are as following:

- Document the impact of globalization on Brahui language and society, - Diagnose the sources of globalization in various sphere of practical life, and Prior to conduct the social survey, secondary resources were explored to know the impact of globalization on Brahui language. In various stages of the study, the impact of globalization on Brahui language were approached to know their point of view, opportunities, and experiences about affects on routine life, language, culture, and environment etc.

A survey was conducted on the watching of programs and dramas of PTV Bolan and other entertainment programs of the electronic media from the students of first and second year of Government Girls College, Khuzdar. The Results shows as under: 
1. $30 \%$ watching the drams of Star Plus

2. $4 \%$ watching the Urdu dramas

3. $6 \%$ watching Geo TV dramas

It is dilemma that the young generation and educated class of society is not interested to watch the dramas in their mother languages.

Another survey conducted in different areas Mastung, Kalat \& Khuzdar and other urban and rural areas of Jhalawan \& Srawan region. Some renowned scholars, teachers, researchers, students and common persons were interviewed on the usage of words of other dominant languages in routine life of people. The Findings revealed that there is an alarming situation due to globalization; electronic gadgets and information technology are gravely having an effect on Brahui language. There are some examples are as under:

1. Names of the relations are Ayee, Luma, Bawa, Tata, Illa, Ambal has been replaced by Mom, Dad, Papa, Aunty, Uncle and Friend.

2. Names of Day i.e., Shambe, Yakshambe, Doshambe, Seshambe, Charshambe, Punchshambe etc has been changed as Saturday, Sunday, Monday, Tuesday, Wednesday and Thursday or Hafta, Atwar, Per, Mungal Budh and Jumarat etc.

3. Indigenous names of girls Mauri, Manguli, Shenlo, Azgend, Gwarikh, Zabad, Beebal, Sehti, Zebul, Shato, Izbutak, Shampeshter, Maurphuzo, Kisankor, Shushang, Marmutak, Matetav etc and names of boys Kaleri, Khati, Jaungu, Khwasti, Barshunki, Mandav, Apursi, Alunji, Batte, Daru, Boro, Mujji, Heezho, Basham, Ambal, Mangal, Pheri, Baburi, Heezahki, Beli, Humi, etc are difficult to find in the Brahui speaking areas.

4. Name of parts of human body Teedar, Maddul klut, altered as Peshani, kopari and Ganja. 
5. They don't know the terms of today, tomorrow, day after tomorrow i.e. Eno, Pagga, Pulme etc and terms of yesterday, day after yesterday i.e. daro, Mulkhudo etc.

6. The people don't know the words of colors i.e. Barbaddi, Borbaddi, Subuk Khesun, Kuben Phushkun, Kuben Gongi and don't pronounce the words Khimishi, Dittri, Jagheri, Ajabbi etc properly.

7. Flood and hurricane are were Haar and let but now called Tufan and Selab. Similarly, Lula and Doli were replaced as pipe and tin.

8. Large scale of names and terminologies of kitchen accessories, food items, and essential items of daily life, dresses and clothing, ornaments, embroideries, livestock, agriculture, environment, social and cultural life has been changed and adopted from English, Persian Arabic and Urdu languages.

\section{Conclusion}

Brahui is one of the oldest languages of the Indian Sub-continent and Brahui speaking people are found in Balochistan, Sindh, Afghanistan, Turkmenistan and Iran. The findings show that Brahui people are living in the region where fast pace of development and other national and international threats of globalization lies i.e., electronic gadgets and information technology growing day by day, which are directly affecting Brahui language. Presently a small scale media i.e., once daily, some weekly, some monthly papers, magazine and journals and some hours programs at PTV Bolan \& Pakistan Broadcasting Corporation and Vsh TV are playing significant role in the promotion of Brahui language but have no sufficient competence to combat the invasion of globalization. There are about 7000 languages spoken in the world and hundreds of languages had been perished due to linguistic reasons. UNESCO has conducted a survey about 3000 dying language of the world and UNESCO Atlas of the World's Languages in Danger declaring that Brahui is a vulnerable language. The survey, interviews and findings are reveal that many Brahui words, names of boys, girls, days, relations, terminologies, names of 
food items, clothing's, embroideries, ornaments, words of routine use of life were replaced and altered by the words of the other dominant languages of electronic media.

\section{References:}

Brahui A. Rehman . 2012, (Second edition) Brahui Ziban o Adab Ki Mukhtasar

Tarikh, Brahui Academy Pakistan, Quetta.

Javed Akhar, 2007, Brahui Lisaniat, Brahui Academy Pakistan, Quetta.

Marri S. Muhammad. 2014, Balochi Ziban o Adab, Ghosa e Adab, Quetta

Mubarak Ali . 2004, Globalization aur Madri Zibanen compiled by Arif

Tabassam, Institute of Development Studies and Practices (IDSP), Pakistan.

Mazhar Arif, 2004, Globalization aur Madri Zibanen compiled by Arif

Tabassam, Institute of Development Studies and Practices (IDSP), Pakistan.

Rehman Tariq Dr. 2004, Globalization aur Madri Zibanen compiled by Arif

Tabassam, Institute of Development Studies and Practices (IDSP), Pakistan.

Siddiqi Khalil. 2000, Ziban Ka Irtaqa, Qalat Printers, Quetta

\section{Webliography}

$\underline{\text { www.brahvilanguage.com }}$

https://.en.wikipedia.org/wiki/Globalization

http://unesdoc.unesco.org/images/0019/001924/192416e.pdf

https://en.wikipedia.org/wiki/Language. 